\title{
The commonplace and unexpected from high energy physicists
}

from David J. Miller

LAST year's wonder has become this year's commonplace. On July 1, the opening day of the International Conference on High Energy Physics at Imperial College, London, five separate new pieces of evidence for neutral weak currents were reported. Moreover, the two groups who had published previously reported greatly improved statistics (see Nature, 245, 119; 1973 and 249,$211 ; 1974$ for discussions of the first neutral-current result, from the Gargamelle bubble chamber at CERN, Geneva and of the early Harvard/Fermi Laboratory results).

The most striking of the new pieces of data comes from a group at the California Institute of Technology, which used an array of counters, spark chambers and magnets in a neutrino beam at the Fermi National Accelerator Laboratory (FNAL). The momentum of their neutrino beam is known better than in the first Harvard-FNAL experiment, and they have a clearer technique for identifying events without fast muons. Their ratio for muonless neutrino events (neutral-current mode) to events with a muon (chargercurrent mode) is 0.22 to 1 . With an antineutrino beam the equivalent ratio is 0.33 to 1 . Although their neutrino beam momenta are around $45 \mathrm{GeV} / c$ or $120 \mathrm{GeV} / c$, compared with around 1 to $10 \mathrm{GeV} / c$ in Gargamelle, this neutrino ratio is very close to the latest Gargamelle figure, and the antineutrino ratio is not alarmingly different, considering the greater uncertainties involved in small antineutrino statistics. A collaboration of 'East Coast' university groups, working at the $29 \mathrm{GeV} / c$ Brookhaven accelerator near New York, has also seen a clear neutral-current signal in a spark chamber experiment.

Two groups reported the observation of neutral-current events with all the final-state particles identified. In Gargamelle and in the big spark chamber arrays, this has been difficult, since the neutrinos interact with the protons or neutrons of a heavy nucleus. At the Argonne Laboratory, near Chicago, a group from Argonne, Concordia College and Purdue University has used the 12-foot bubble chamber, filled with liquid hydrogen or deuterium, to identify about 14 events in which a neutrino struk a single proton (or a neutron in deuterium). The final state in each of these events contained a positive or a neutral pion, plus a recoil neutron or proton (and also a visible 'spectator' proton, in deuterium). As with all neutral-current experiments, the final-state neutrino in each event was not observed. The numbers are too small, to date, to do more than to establish the existence of these processes. Experimenters from CERN obtained similar results by studying neutrino interaction on the free protons in propane, using film which was taken 7 or 8 years ago, before the existence of neutral currents was even suggested. It demonstrates that there should be a great deal of useful data to be obtained from the next Gargamelle run, with a propane filling and a more intense neutrino beam.

There can now be no doubt that neutrinos and antineutrinos interact with nucleons (that is, protons and neutrons) in two distinct ways. The charged-current mode is more copious, but the neutral-current mode, in which another neutrino goes off afterwards rather than a charged muon, is well established. It is even becoming possible to put realistic limits on the 'Weinberg angle', a parameter which governs the relative strength of the charged and neutral currents in the simplest unified theory of weak and electromagnetic interactions.

One interesting question still awaits a definite answer; that is, how do neutrinos and antineutrinos interact with electrons? The Gargamelle collaboration reported the observation of two events in which an electron appears to have been produced by an antineutrino interaction. Although the calculated background is small, there can be no certainty in the observation of such a small signal. But the rate is roughly what would be expected within the framework of the simplest theory, using a value for the Weinberg angle which is consistent with the neutrino-nucleon experiments. This is the first experimental evidence that the neutral neutrino current might interact with electrons as well as with protons.

\section{Muon puzzles}

There was speculation at the meeting about both new elementary particles and new interactions. One new effect is the direct production of charged leptons from hadron collisions. The leptonselectron, muon and neutrino-have never been observed to take part in strong interactions. The hadrons-the proton and neutron, the hyperons, the mesons and all of the resonant statesare the only known strongly interacting particles. They were thought to produce leptons only in weak or electromagnetic decays, not in their strong collisions.

A group from CERN, Columbia and Rockefeller Universities in New York, and from Saclay in France, working at the CERN Intersecting Storage Rings in Geneva, has now found convincing evidence that electrons are directly produced in proton-proton collisions at the very highest observed energies. Another group from Columbia, working at the Fermi National Laboratory near Chicago, has seen the same sort of effect, with both electrons and muons coming from proton-proton collisions at somewhat lower energies. A Chicago, Harvard, Pennsylvania and Wisconsin group also has seen direct muon production at the Fermi Laboratory, and direct muon production has been reported from the even lower energy accelerators at Serpukhov in the Soviet Union and at Brookhaven, New York. All of these data have, of course, been corrected for indirect muon production by means of the weak decays of mesons. In every case the ratio of direct lepton production to pion production is about 1 to 10,000 . The ratio does not vary much over the large energy span of these different machines. It also seems roughly constant as the transverse component of the lepton momentum, perpendicular to the incoming protons, is varied from 2 to $5 \mathrm{GeV} / c$.

Two sorts of explanation have been suggested for direct lepton production; the revolutionary or the merely surprising. One revolutionary explanation requires that 'charmed' particles are being produced, and decaying rapidly to normal particles including muons or electrons. 'Charm' is a postulated new quantum number, similar to the old-established 'strangeness' quantum number. It is needed to explain, among other things, the absence of strange-particle production in neutral-current weak interactions. The merely surprising explanation requires that most of the pion production in high energy proton-proton collisions actually comes from the production of massive resonances which decay to pions by the strong interaction. Some of these resonances, in particular the 'rho', the 'omega' and the 'phi' known as the 'vector mesons', also decay occasionally by means of the electromagnetic interaction to a pair of electrons or a pair of muons. Preliminary calculations, using vector meson decays, give a lepton to pion ratio of about 1 to 100,000 . Nobody knows yet whether the extra factor of 10 can be found to make this calculation match the observed rate. But even if the factor can be found, and a revolutionary explanation is not needed, ideas of panticular production at high energies will have been radically changed.

The Fermi Laboratory and Harvard 
neutrino experiment has revealed two puzzling muon events of quite a different kind. Each seems to be the result of the interaction of a $150-\mathrm{GeV}$ neutrino in their apparatus, producing two muons and a shower of hadrons. Ordinary 'charged-current' neutrino interactions contain one final-state muon, and neutral-curient events have no muons. As a theorist remarked at the conference: "taking preliminary results seriously is a well known way of making a fool of yourself". Nevertheless, there is much speculation that these dimuons could be due to the decay of a totally new kind of particle. Some say 'charmed' particles again; some say it may be the 'intermediate boson' that carries the weak force, a sort of heavy photon; some say it is a heavy lepton of the kind needed in the unified weak and electromagnetic theory of Georgi and Glashow. Certainly, if this observation is correat, then no 'merely surprising' explanation will do.

\section{Prospecting for a dead slab}

\section{from Peter J. Smith \\ Geomagnetism Correspondent}

LITHOSPHERIC slabs descending beneath trench systems are known to have seismic velocities which are measurably higher than those in the surrounding mantle. Because such downthrusting slabs can be recognised in simpler and more convenient ways, not least by their deep seismicity, there may be little need to use the seismic velocity property to detect Benioff zones which are still active. But what about zones in which subduction has now ceased and which are seismically quiet or dead? When subduction ceases, the relevant slab, initially cooler than the mantle into which it was previously descending, will slowly warm until the thermal and compositional contrast between it and the surrounding mantle disappears; but until this process is complete the 'dead' slab may retain enough of its identity to maintain the velocity contrast. So can this contrast be used to detect the slab's presence?

That there are likely to be recently dead slabs to detect may be inferred by extrapolating known plate tectonic processes backward in time. The evolution on the western margin of North America is a good case in point and probably the most-studied example. Magnetic anomalies in the north-east Pacific suggest that a Farallon plate (sometimes called the Juan de Fuca plate or the Gorda plate) descended along the boundary between it and the North American plate until about 30 million years ago, at which time a spreading ridge between the Farallon and Pacific plates began to collide with the western North America trench and strike-slip motion commenced along the San Andreas fault. In this way it is thought, the plate boundary along western North America began to to change from a subduction zone to a transform fault. There is evidence from seismic reflection studies of the continental margin, from the occurrence of subcrustal earthquakes, from andesitic volcanism in the Cascades and from marine magnetic anomalies that north of the Mendocino fracture zone subduction may still be occurring very slowly. South of Cape Mendocino, however, subduction has ceased, although Atwater (Bull. Geol. Soc. Am., 81, 3513; 1970) has concluded that the cessation may have occurred no more than a few million years ago.

The implication here is that the remains of the Farallon slab may still lie beneath California and may still have sufficient of its original identity to produce a measurable velocity contrast. To test whether this is indeed so, Solomon and Butler (Earth planet. Sci. Lett., 21, 421 ; 1974) have attempted to detect such a contrast by analysing the traveltime delays of teleseismic $P$ waves in the region. The method adopted was derived from that used by Davies and McKenzie (Geophys. J., 18, 51; 1969) to show that shallow earthquakes and explosions above a descending slab produce distinctive patterns of travel-time residuals for teleseismic $P$ waves as a result of the velocity anomaly within the slab. The basic residuals obtained by Solomon and Butler were the travel-time delays at each station concerned for $\mathbf{P}$ waves from many different earthquakes and explosions. But to remove uncertainties in source locations and origin times and to reduce nearsource contributions to the total traveltime residuals, the residuals actually plotted were the differences between the travel-time delays at each station and the corresponding delays at a reference station overlying what is presumed to be more uniform mantle. The sources were mid-plate earthquakes and explosions, deep earthquakes, and "carefully screened" (to avoid obvious near-source heterogenities) earthquakes on spreading centres and transform faults.

In the absence of any detailed knowledge of the position and extent of the supposed dead slab, the stations chosen were those between latitudes $37^{\circ} \mathrm{N}$ and $50^{\circ} \mathrm{N}$ and between longitudes $116^{\circ} \mathrm{W}$ and $125^{\circ} \mathrm{W}$ at which $\mathrm{P}$ wave arrivals had been regularly reported during the period 1964-1970 (a selection process which eliminated all but seven stations, three of which were regarded as reference stations). The residuals obtained from various station bairs were plotted on residual-spheres, the resulting plots being a measure of the variation of travel-time delay with direction of wave propagation in the upper mantle beneath the relevant station.

The residual-sphere for station LON in the Cascades in Washington (referenced to BMO, Blue Mountain Observatory to the east) failed to indicate any systematic trends in residuals. If a high velocity slab lies in the upper mantle beneath western Washington and if its upward projection cut the Earth's surface near LON, an eastward-dipping band of negative residuals (early arrivals) might be expected. No such band was observed. But for station pairs based on the three primary stations in California (MIN in the southern Cascades, ORV and JAS along the western edges of the Sierra Nevada), a group of consistently negative residuals was observed towards the east in each case. Moreover, the average travel-time advance in the eastern quadrants of the residual-spheres (calculated in the same way for each sphere) decreased southwards from $1.2 \mathrm{~s}$ for MIN, through $0.8 \mathrm{~s}$ for ORV, to $0.5 \mathrm{~s}$ for JAS.

These patterns of travel-time residuals (irrespective of magnitude) are consistent with the presence of a dead slap dipping eastwards beneath California. On the other hand, they cannot be said to prove the point absolutely because the same data could in principle be explained using a model with undulations in the depth to the top or bottom of the low velocity zone. Moreover, the data are not as complete as they might be because of a paucity of seismic sources in eastern North America. But seen in the light of other evidence the case for a dead slab is more convincing. In addition to the plate tectonic inferences already quoted, support may be adduced from heat flow studies, for example. According to Roy et al. (in The Nature of the Solid Earth, McGraw-Hill, 1972), the low heat flow in the Sierra Nevada apparently requires a heat sink in the shallow $(\sim 50 \mathrm{~km})$ mantle--a role that could easily be filled by the postulated slab. If this interpretation is accepted, the implication then is that between the Pacific plate and the Sierra Nevada the Farallon plate dips at an angle of perhaps no more than $10^{\circ}-15^{\circ}$. A more detailed analysis of the travel-time data, on the other hand, suggests that east of the Sierra Nevada the dip is much more steep (possibly $40^{\circ}-50^{\circ}$ ).

The southward decrease in the magnitude on the travel-time advance could result from differences in the positions of the stations with respect to the position on the Earth's surface of the upward projection of the supposed slab, or it could be due to a real southward decrease of the $P$ wave velocity in the slab. Solomon and Butler favour the latter explanation on the grounds that the three stations are similarly 